\section{Menstruating primary umbilicus cutaneous endometriosis: A case report and review of literature}

\author{
Idowu Pius Ade-Ojo* and Oluwadare Martins Ipinnimo
}

Obstetrics \& Gynaecology, Ekiti State University, Nigeria

\section{Abstract}

Primary umbilical cutaneous endometriosis is a rare umbilical endometrioma that affects women who are within the reproductive age group. It may be associated with infertility and severe dysmenorrhea and can be difficult to diagnosed in an asymptomatic patient.

We report a case of a 38-year-old nulliparous with seven years history of infertility and severe dysmenorrhea. Her hormonal profile assay and hysterosalpingogram results were normal while her husband semen analysis was also within normal range. She complained of monthly bleeding from a painful rubbery multilobate cutaneous nodule on the umbilicus of one year duration. She was diagnosed of cutaneous endometriosis. The diagnosis was confirmed histologically and she had surgical excision with good outcome.

\section{More Information}

*Address for Correspondence: Idowu Pius Ade-Ojo, Senior Lecturer, Obstetrics \& Gynaecology, Ekiti, State University, Nigeria, Email: ipadeojo@eksu.edu.ng

Submitted: April 27, 2021

Approved: May 04, 2021

Published: May 05, 2021

How to cite this article: Ade-Ojo IP, Ipinnimo OM. Menstruating primary umbilicus cutaneous endometriosis: A case report and review of literature. Clin J Obstet Gynecol. 2021; 4: 069-071.

\section{DOI: 10.29328/journal.cjog.1001090}

Copyright: ๑ 2021 Ade-Ojo IP, et al. This is an open access article distributed under the Creative Commons Attribution License, which permits unrestricted use, distribution, and reproduction in any medium, provided the original work is properly cited.

Keywords: Cutaneous endometriosis; Endometriosis; Infertility

Check for updates

open Access

\section{Introduction}

First described by a German Physician Daniel Schroen in 1690, the term endometriosis defines the presence of endometrial glands and stroma outside the uterine cavity $[1,2]$. Except on the spleen, endometriotic lesion could occur on nearly all organs and mucosa surfaces in the body including the brain and extremities [1-5]. Although it affects about 15\% of women in their reproductive age [1], it is very rarely seen before menarche [6] and in postmenopausal women [7]. The ovaries in $88 \%$ of cases bear the burden of endometriosis, Fallopian tube are also proportionately commonly involved [1].

Cutaneous endometriosis described the type of endometriosis found in the skin and subcutaneous tissues and account for $0.03 \%$ to $0.15 \%$ of cases [8]. There are two forms of cutaneous endometriosis; primary or spontaneous typically arising from the umbilicus (30\%) [1] and secondary or cicatricial which usually follows procedure involving the uterus (70\%) [1], the cause of primary cutaneous endometriosis is unclear but secondary cutaneous endometriosis is believed to occur due to implantation of endometrial micro seedlings from abdominal and pelvic surgery particularly involving the uterus [1]. We present a case of 38-year-old nullipara with one year history of cyclically bleeding umbilicus who we were managing for primary infertility. We also present the review of relevant literature to further clarify salient issues concerning this rare but important disorder.

\section{Case description}

A 38-year-old nulliparous who presented to us on the $2^{\text {nd }}$ day of her menstruation with 7 years history of inability to conceive and severe dysmenorrhea. Her hormonal assay results, hysterosalpingogram and her husband's semen analysis were all normal. She noticed that she developed a small painful umbilical swelling about a year ago which increased in size and bled every month whenever she observed her monthly menstrual flow.

On examination, we found a darker than her skin tender multilobate nodule on her umbilicus with erythema of the overlying skin and blood trickling down from the center of the mass. We suspected an umbilical cutaneous endometriosis and a biopsy of the lesion was taken and samples sent for histology. The result of histology is as follows "Specimen received was a grayish white tissue with section of skin tissue and an unremarkable epidermis, scattered within the dermis are numerous capillary and foci of benign endometrial glands and stroma with area of hemorrhages". The diagnosis of primary umbilical cutaneous endometriosis was made. She had an ultrasound scanning done which was not remarkable. A diagnostic laparoscopy was also done which ruled out concomitant intrapelvic endometriosis.

She had the multilobate nodule excised under general anesthesia and it showed contiguity with the peritoneum. There were no postoperative sequelae (Figure 1). 


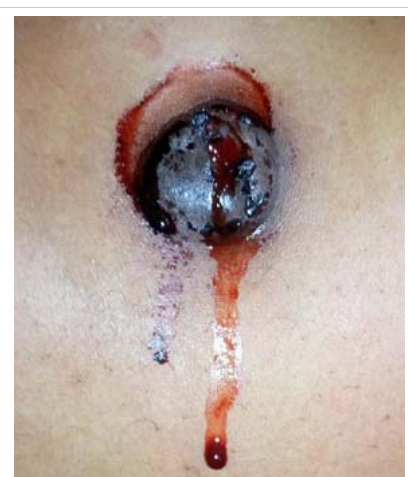

Figure 1: Showing an image of the menstruating umbilicus of a 38-year-old nullipara who presented at the Gynecological clinic of the Ekiti State University Teaching Hospital on the $2^{\text {nd }}$ day of her menstrual flow.

\section{Discussion}

Primary or spontaneous cutaneous endometriosis appear in absence of surgery and are usually located in the umbilicus as found in our patient, it could also be found in the inguinal and abdominal walls [8]. Cutaneous endometriosis in the umbilicus also known as villar nodules, with reference to the person that first describe the lesion in 1886 [1]. It manifests as a rubbery or firm nodule ranging in size from some $\mathrm{mm}$ to 6-9 cm (mean 2-2.5 cm) [1]. Its color varies from red, blue to brown-black, depending on the amount of hemorrhage and the penetration depth of ectopic endometrial tissue. Occasionally, the nodule is flesh-colored [3]. It is usually single and often multilobate, although multiple discrete nodules may be present [3]. Clinical features include pain, bleeding, swelling, tenderness and growth correlated with the menstrual cycle. However, not all symptoms are present in a given patient and some patients are totally asymptomatic [1].

The diagnosis of cutaneous endometriosis could be suspected clinically. Other diagnostically helpful imaging methods include Dermatoscopy, Magnetic Resonance Imaging, and Ultrasonography. Ultrasonography and Computed Tomography are more accessible than but not as sensitive as Magnetic Resonance Imaging. Fine needle aspiration cytology has been used, but its results may be inconclusive. Serum CA125 levels may be increased (up to $260 \mathrm{U} / \mathrm{ml}$ ), but this finding is not specific for cutaneous endometrosis [1].

The treatment of cutaneous endometriosis is mainly surgical, preferably performed at the end of the menstrual cycle when the lesion is small in order to achieve a minimal excision [8]. The technique of removal varies depending on the size and extent of the lesion, from simple excision with wide margins under local anesthesia to laparoscopic excision en block of the umbilicus [8]. Treatment with GonadotropinReleasing Hormone agonists, danazol, and contraceptive pills can be given in order to reduce tumor size before excision, or provide relief from the symptoms; these are insufficient as sole treatments and may lead to incomplete excision [2].

Gynecologic examination and hormonal evaluation are recommended after excision of cutaneous endometriosis in order to detect an associated pelvic endometriosis. Abdominal and transvaginal ultrasonography or MRI scan should be performed in all asymptomatic patients, but whether laparoscopic examination should be systematically performed is still debated [1].

The prognosis of cutaneous endometriosis is good. Recurrences are uncommon if excision is performed with clean and wide margins. However, malignant transformation had been reported in $0.3 \%$ to $1 \%$ of scar endometriosis and should be suspected in the case of rapidly-growing or recurrent lesions [9]. The commonest histological type is clear cell $(66.7 \%)$ followed by endometroid $(14.6 \%)$, serous $(8.5 \%)$, mixed (4.2\%) and others (6.2\%) [10].

Cutaneous endometriosis is rare and has not been extensively reported. This is the first time that this rare disorder is being reported from our center. Our patient was diagnosed with cutaneous endometriosis based on the presence of endometrial glands and stroma on the identified lesion.

The differential diagnosis of umbilical cutaneous endometriosis includes lesion located on the umbilicus like keloid, hypertrophic scar, dermatofibroma and cutaneous metastasis of cancers [Sister Mary Joseph nodule] and dermatofibrosarcoma protuberance, but all these lesions don't present with cyclical bleeding from the umbilical lesion like observed in this patient.

Once the diagnosis of cutaneous endometriosis has been made the definitive management is excision biopsy with wide margin. Other forms of treatment include the use of hormonal therapy such as combine oral contraceptive pills and gonadotropin-releasing hormone agonist which was not considered in this patient due to the history of infertility at presentation. Most of this drug act by preventing proliferation of the endometrium.

Prognosis is considered favorable especially when surgical excision is considered as done in this patient. Recurrence rate following surgical excision is $9 \%$ [3] which put this management option as a reasonable option.

In conclusion, physicians should consider cutaneous endometriosis in the differential diagnosis of nodules appearing over the abdominal wall, especially the umbilicus, in reproductive-age women. The diagnosis can be suspected clinically based on history, but should be confirmed with histological examination. Treatment is surgical excision with wide margins. Gynecologic evaluation is recommended after excision, especially if the patient is symptomatic, for pelvic endometriosis. Malignant transformation, although very rare, should be suspected in recurrent or rapidly growing lesions. 


\section{References}

1. Kyamidis K, Lora V, Kanitakis J. Spontaneous cutaneous umbilical endometriosis: report of a new case with immunohistochemical study and literature review. Dermatol Online J. 2011; 17: 5. PubMed: https://pubmed.ncbi.nlm.nih.gov/21810390/

2. Buescher E, Nezhat C. Endometriosis: Diagnosis and Treatment: From Fingernails to the Robot. Cureus. 2012.

3. Ichida M, Gomi A, Hiranouchi N, Fujimoto K, Suzuki K, et al. A case of cerebral endometriosis causing catamenial epilepsy. Neurology. 1993; 43: 2708-2709.

PubMed: https://pubmed.ncbi.nlm.nih.gov/8255485/

4. Giangarra C, Gallo G, Newman R, Dorfman H. Endometriosis in the biceps femoris. A case report and review of the literature. J Bone Joint Surg. 1987; 69: 290-292.

PubMed: https://pubmed.ncbi.nlm.nih.gov/3805095/

5. Mehdi K, Kumar A. Spontaneous cutaneous endometriosis in Mons Pubis: review of literature. Med J Armed Forces India. 2021.
6. Marsh E, Laufer M. Endometriosis in Premenarcheal Girls Who Do Not Have an Associated Obstructive Anomaly. Fertil Steril. 2005; 83: 758-760.

7. Manero M, Royo P, Olartecoechea B, Alcázar J. Endometriosis in a postmenopausal woman without previous hormonal therapy: a case report. J Med Case Rep. 2009; 3.

8. Fernández-Aceñero $\mathrm{M}$, Córdova $\mathrm{S}$. Cutaneous endometriosis: review of 15 cases diagnosed at a single institution. Arch Gynecol Obstet. 2010; 283: 1041-1044.

PubMed: https://pubmed.ncbi.nlm.nih.gov/20422419/

9. Daisley jr. H, Trim S, Daisley A. Caesarean section scar endometriosis: A case report and review of the literature with special emphasis on malignant transformation. Our Dermatology Online. 2018; 9: 176-179.

10. Mihailovici A, Rottenstreich M, Kovel S, Wassermann I, Smorgick N, et al. Endometriosis-associated malignant transformation in abdominal surgical scar: A PRISMA-compliant systematic review. Medicine (Baltimore). 2017; 96: e9136.

PubMed: https://pubmed.ncbi.nlm.nih.gov/29245355/ 